\title{
Impact of impaired insulin secretion and insulin resistance on the incidence of diabetes in a Japanese cohort. Reply to Yamauchi $K$ and Aizawa $T$ [letter]
}

\author{
Akiko Morimoto • Yukako Tatsumi • Kijyo Deura • \\ Naomi Miyamatsu • Mitsuhiko Noda • Shaw Watanabe
}

Received: 2 September 2013 / Accepted: 5 September 2013 /Published online: 21 September 2013

(C) Springer-Verlag Berlin Heidelberg 2013

Keywords Epidemiology · Insulin resistance · Insulin secretion · Japan · Type 2 diabetes

\begin{tabular}{ll}
\multicolumn{2}{l}{ Abbreviations } \\
FPG & Fasting plasma glucose \\
HOMA-IR & HOMA of insulin resistance \\
i-IIS & Isolated impaired insulin secretion \\
IIS & Impaired insulin secretion \\
i-IR & Isolated insulin resistance \\
IR & Insulin resistance \\
PAF & Population-attributable fraction
\end{tabular}

To the Editor: We appreciate the comments from Dr Yamauchi and Dr Aizawa [1] on our recent publication in

\footnotetext{
A. Morimoto $(\bowtie) \cdot$ Y. Tatsumi $\cdot N$. Miyamatsu Department of Clinical Nursing, Shiga University of Medical Science, Tsukinowa-cho Seta, Otsu, Shiga 520-2192, Japan e-mail: aki62@belle.shiga-med.ac.jp

Y. Tatsumi

Department of Mathematical Health Science, Graduate School of Medicine, Osaka University, Osaka, Japan

K. Deura

Saku Central Hospital, Nagano, Japan

M. Noda

Department of Diabetes Research, National Center for Global Health and Medicine, Tokyo, Japan

S. Watanabe

Life Science Promoting Association, Tokyo, Japan
}

Diabetologia [2]. We sincerely respond to their concerns as follows.

Pathophysiological risk and population-based risk of impaired insulin secretion (IIS) and insulin resistance (IR) First of all, our investigation was an epidemiological study to clarify the population-based risk, and our results only suggest a certain pathophysiological mechanism as a target of future experimental and clinical research. Our study simply showed predicted values for the incidence of type 2 diabetes for certain clinical findings, such as isolated impaired insulin secretion (i-IIS) or isolated insulin resistance (i-IR) as defined in our paper, measured on a single occasion. In an additional analysis conducted for the present letter, the multivariable-adjusted HR for the incidence of type 2 diabetes was 1.69 (95\% CI 1.17, 2.42) in the i-IIS group compared with the i-IR group after adjustment for age, sex, family history of diabetes, current smoking, alcohol consumption and exercise. After adjustment for the above-mentioned variables and BMI, waist circumference, body fat, systolic BP, HDL-cholesterol, $\log _{e}$-transformed triacylglycerol, $\log _{e}$-transformed $\gamma$-glutamyltransferase, uric acid, high-sensitive C-reactive protein and fasting plasma glucose (FPG), the multivariable-adjusted HR for the incidence of type 2 diabetes was 3.25 (95\% CI $2.15,4.90)$ in the i-IIS group compared with the i-IR group.

Estimation of IR As mentioned by Yamauchi and Aizawa [1], estimation of IR by calculating the HOMA-IR, which primarily reflects hepatic IR [3], was one of the major limitations of our study. We are planning to measure serum insulin concentrations at 0 (fasting), 30, 60 and 120 min using standard $75 \mathrm{~g}$ OGTTs, and we will estimate the risk of whole body IR in the near future. 
Table 1 Incidence rates, HRs and PAFs for the development of type 2 diabetes, according to baseline IIS and IR status: additional analysis

\begin{tabular}{|c|c|c|c|c|}
\hline Variable & Normal $(n=1,550)$ & i-IIS $(n=900)$ & $\mathrm{i}-\mathrm{IR}(n=505)$ & IIS plus IR $(n=104)$ \\
\hline Incident cases ( $n /$ person-years) & $24 / 5,447$ & $126 / 3,032$ & $39 / 1,748$ & $30 / 326$ \\
\hline Incidence rate (/1,000 person-years) & 4.4 & 41.6 & 22.3 & 92.0 \\
\hline \multicolumn{5}{|l|}{$\operatorname{HR}(95 \% \mathrm{CI})$} \\
\hline Model 1 [2] & 1.0 & $8.27(5.33,12.83)$ & $4.90(2.94,8.17)$ & $16.93(9.80,29.25)$ \\
\hline Model 2 & 1.0 & $6.19(3.95,9.70)$ & $1.91(1.18,3.29)$ & $4.39(2.43,7.93)$ \\
\hline \multicolumn{5}{|l|}{ PAF $(95 \% \mathrm{CI})^{\mathrm{a}}, \%$} \\
\hline Model 1 [2] & - & $50.6(46.7,53.0)$ & $14.2(11.8,15.6)$ & $12.9(12.3,13.2)$ \\
\hline Model 2 & - & $48.2(43.0,51.6)$ & $8.5(2.8,12.4)$ & $10.6(8.1,12.0)$ \\
\hline
\end{tabular}

Normal, insulinogenic index $>51.7$, HOMA-IR $\leq 1.6$; i-IIS, insulinogenic index $\leq 51.7$, HOMA-IR $\leq 1.6$; i-IR, insulinogenic index $>51.7$, HOMA-IR $>1.6$; IIS plus IR, insulinogenic index $\leq 51.7$, HOMA-IR $>1.6$

Model 1, adjusted for age, sex, family history of diabetes (yes or no), current smoking (yes or no), alcohol consumption ( $0 \mathrm{~g} / \mathrm{week}, 1-139 \mathrm{~g} / \mathrm{week}$ or $\geq 140 \mathrm{~g} /$ week) and exercise ( $0 \mathrm{~min} / \mathrm{week}, 1-119 \mathrm{~min} /$ week or $\geq 120 \mathrm{~min} / \mathrm{week}$ )

Model 2, adjusted for all factors in Model 1 plus BMI, waist circumference, body fat, systolic BP, HDL-cholesterol, $\log _{e}$-transformed triacylglycerol, $\log _{e}$-transformed $\gamma$-glutamyltransferase, uric acid, high-sensitive $\mathrm{C}$-reactive protein and FPG

${ }^{\text {a }}$ PAF (\%) was calculated using multivariable-adjusted HR (Models 1 or 2)

Other confounding factors in the Cox model The results of additional analysis are shown in Table 1. Age, sex, family history of diabetes, current smoking, alcohol consumption and exercise were included in Model 1 [2]; and BMI, waist circumference, body fat, systolic BP, HDLcholesterol, $\log _{e}$-transformed triacylglycerol, $\log _{e^{-}}$ transformed $\gamma$-glutamyltransferase, uric acid, high-sensitive C-reactive protein and FPG were included in Model 2. The results in Model 2 in Table 1 indicate that i-IIS and i-IR are pathophysiological risk factors for type 2 diabetes after full adjustment, and the PAF (48.2\%) of type 2 diabetes onset due to i-IIS was similarly high.

We did not adjust for BMI, waist circumference, FPG, etc. for two reasons. First, IR is a syndrome associated with the clustering of metabolic disorders, including obesity, hypertension, lipid abnormalities and atherosclerotic cardiovascular disease [4]. Obesity, in particular, would tend to lead to type 2 diabetes, mainly through IR [5]. Therefore, adjustment for metabolic disorders, especially BMI and waist circumference, may be an over-adjustment. This may result in an underestimation of the risks of IR on type 2 diabetes. Second, we assessed the population-based risk of the state of being insulin resistant (i.e. individuals with IR have high BMI, waist circumference, FPG, etc.) on the incidence of type 2 diabetes.

Minimum value of the insulinogenic index Yamauchi and Aizawa mention that negative values cannot be used to calculate the insulinogenic index. However, there were no negative values and the minimum insulinogenic index in the individuals eligible for our analysis was $2.8 \mathrm{pmol} / \mathrm{mmol}$. Accordingly, noone was excluded from the analysis for this reason.

Acknowledgements We thank T. Okamura (the Department of Preventive Medicine and Public Health, Keio University, Tokyo, Japan) for his advice on this paper.

Funding A. Morimoto was the recipient of a Grant-in-Aid for Japan Society for the Promotion of Science Fellowship. This study was funded by grants from the Ministry of Health, Labor and Welfare, Japan.

Duality of interest The authors declare that there is no duality of interest associated with this manuscript.

Contribution statement All authors contributed to drafting or critical revision of the letter. All authors approved the final version of the letter.

\section{References}

1. Yamauchi K, Aizawa T (2013) Impact of impaired insulin secretion and insulin resistance on the incidence of diabetes in a Japanese cohort. Diabetologia. doi:10.1007/s00125-013-3046-2

2. Morimoto A, Tatsumi Y, Deura K et al (2013) Impact of impaired insulin secretion and insulin resistance on the incidence of type 2 diabetes mellitus in a Japanese population: the Saku study. Diabetologia 56:1671-1679

3. Tripathy D, Almgren P, Tuomi T, Groop L (2004) Contribution of insulin-stimulated glucose uptake and basal hepatic insulin sensitivity to surrogate measures of insulin sensitivity. Diabetes Care 27:2204-2210

4. DeFronzo RA, Ferrannini E (1991) Insulin resistance. A multifaceted syndrome responsible for NIDDM, obesity, hypertension, dyslipidemia, and atherosclerotic cardiovascular disease. Diabetes Care 14:173-194

5. Kahn SE, Hull RL, Utzschneider KM (2006) Mechanisms linking obesity to insulin resistance and type 2 diabetes. Nature 14:840-846 